\title{
Prevalence and Correlation of Metabolic Syndrome: A Cross-Sectional Study of Nearly 10 Million Multi-Ethnic Chinese Adults
}

This article was published in the following Dove Press journal: Diabetes, Metabolic Syndrome and Obesity: Targets and Therapy

\author{
Yinxia $\mathrm{Su}^{1,2, *}$ \\ Yaoqin Lu (iD) ${ }^{1,3, *}$ \\ Wenli $\mathrm{Li}^{\mathrm{l}} *$ \\ Mingyue $\mathrm{Xue}^{2,4}$ \\ Chen Chen $\left(10^{5}\right.$ \\ Muyaseer Haireti ${ }^{1}$ \\ Yuanyuan $\mathrm{Li}^{1}$ \\ Zhenhui Liu ${ }^{6}$ \\ Yanshi Liu ${ }^{6}$ \\ Shuxia Wang ${ }^{2}$ \\ Hua Yao (iD) ${ }^{2}$
}

'School of Public Health, Xinjiang Medical University, Urumqi, Xinjiang, People's Republic of China; ${ }^{2}$ Health Management Institute, Xinjiang Medical University, Urumqi, Xinjiang, People's Republic of China; ${ }^{3}$ Center of Disease Control and Prevention, Urumqi, Xinjiang, People's Republic of China; ${ }^{4}$ The Fourth Affiliated Hospital of Xinjiang Medical University, Urumqi, Xinjiang, People's Republic of China; ${ }^{5}$ School of Biomedical Sciences, University of Queensland, Brisbane 4072, Australia; ${ }^{6}$ Department of Microrepair and Reconstruction, The First Affiliated Hospital of Xinjiang Medical University, Urumqi, Xinjiang, People's Republic of China

*These authors contributed equally to this work
Purpose: This study aimed to examine the prevalence and correlates of metabolic syndrome (MetS) in multi-ethnic populations of Northwest China based on Large-scale provincial health checking data.

Patients and Methods: A total of 9,745,640 Chinese aged $\geq 18$ years in Xinjiang, the largest autonomous region of multi-ethnic in China, were enrolled from Feb. to Sep. 2019. MetS was defined by modified Adult Treatment Panel (ATP III) criteria.

Results: The overall prevalence of MetS was 20.85\% [Sex: 20.06\% female, $21.56 \%$ male; Age: $39.22 \% 60$-years and above, $26.32 \% 40$ - to 59-years and 9.56\% 18- to 39-years; Ethnicity: $28.19 \%$ Hui followed by Han (26.39\%), Uyghur (18.56\%), Other (18.61\%), Kazak (17.98\%), Mongolian (17.87\%), Kyrgyz (14.44\%)]. People living in cities and towns $(23.03 \%)$ or North $(24.78 \%)$ had higher prevalence of MetS than rural $(19.94 \%)$ and South (17.66\%). Although the prevalence of hypertension, smoking, drinking and lack of physical activity were higher in Kyrgyz, Mongolian and Kazak than those of other ethnic groups, the risk of MetS was lower.

Conclusion: The results indicated that a high prevalence of MetS in Hui and Han groups and people living in cities and towns. Living in North Xinjiang and elder population tend to have higher prevalence of MetS. The prevention and management of MetS in these populations should be prioritized.

Keywords: metabolic syndrome; MetS, Chinese, ethnic groups, adults, prevalence

\section{Introduction}

Metabolic syndrome (MetS) is caused by combined effects of multiple metabolic risk factors including central obesity, impaired sugar control, dyslipidemia, hypertension, insulin resistance (IR), hyperuricemia and microalbuminuria. ${ }^{1}$ Patients with MetS are at increased risk for developing type 2 diabetes mellitus, cardiovascular diseases, and other chronic diseases with high mortality and morbidity. ${ }^{2}$ A close correlation between MetS and increased risk of chronic diseases (e.g., cardiovascular disease, type 2 diabetes, arthritis, chronic kidney disease, schizophrenia) with short life-span have been reported. ${ }^{2-4}$

It is estimated that about $20-30 \%$ of the world's population suffer from MetS, and the prevalence is on the rise. ${ }^{5}$ The prevalence of MetS varies between different regions. Based on the National Cholesterol Education Program data (NCEP) Adult Treatment Panel III (ATP III), the prevalence of MetS in $\geq 20$-year-old adults in the United States was $34.7 \%{ }^{6}$ whereas in Canada, it was nearly $25 \%$ in multi-ethnic
Correspondence: Hua Yao; Shuxia Wang Email yaohua0I@sina.com;

2724443591@qq.com 
adult population $;^{7}$ and in sub-Saharan Africa, it was $17.1 \%$ in multi-ethnic adult population. ${ }^{8}$ In Australia, the prevalence of MetS was $21.7 \%$ in adults $\geq 25$-years-old in a national wide survey. ${ }^{9}$ The prevalence of MetS in Indonesia was $21.66 \%$ among provinces and ethnic groups. ${ }^{10}$ The latest national meta-analysis in 2016 estimated the prevalence of MetS in China as $24.5 \%,{ }^{11}$ but multi-ethnic population here aged $\geq 20$ years showed a lower prevalence of MetS (15.9\%) in the study. ${ }^{12}$ As to mono-population, $30.0 \%$ prevalence of MetS was observed in Han ethnic group aged $\geq 18$ years in Zhejiang province, China, ${ }^{13}$ and a study in the Han group in northeast China reported an overall prevalence of MetS as $41.3 \% .{ }^{14}$ It is clear that the prevalence of MetS in the mono-population higher than that in the multiethnic population and the Chinese Han group is much higher than in other ethnic minorities in China. This is worth exploring the underlying reasons. In addition, MetS components such as hypertension, high blood sugar and dyslipidemia are major and modifiable risk factors for major chronic diseases. It is important to track the difference in MetS prevalence in different ethnic groups in order to obtain an insight into the occurrence of MetS, and to explore new risk factors. Recent studies of MetS in different regions and different populations of China were performed with different diagnosis standards, ${ }^{15-18}$ and the results were inconsistent with small sample sizes, short age spans and different regional factors. Big data epidemiological analysis of risk factors of MetS in multi-ethnic groups in same region with same diagnosis standard is urgently needed.

Since the proportion and distribution of body fat in Asians are different from those in North America and Europe, ${ }^{19,20}$ the definition of obesity applicable to the Western population may not fully apply to the Asian population. The Harmonized criteria have defined cutoffs for Asian central obesity (waist circumference: $\geq$ $90 \mathrm{~cm}$ for males; $\geq 80 \mathrm{~cm}$ for females) and reduced cutoff for Asian hyperglycemia ( $\geq 5.6 \mathrm{mmol} / \mathrm{L}$, instead of 6.1 $\mathrm{mmol} / \mathrm{L}$ in the NCEP-ATP III). Modified Adults Treatment of Obesity Panel (ATP III) criteria were reported as the most suitable for Asian populations. ${ }^{21,22}$ The prevalence of MetS depends on a series of non-modifiable genetic (gender, age, ethnicity) and modifiable (lifestyle, diet) risk factors. These factors are known to, directly or indirectly, influence MetS among the populations. Studies indicated the significant but inconsistent influence of lifestyle habits on the prevalence. ${ }^{23}$ The relationship of lifestyle behaviors and MetS among major ethnicities were not evaluated in some studies. ${ }^{24,25}$ Contribution of non-modifiable factors in some groups to MetS prevalence cannot be completely excluded.

To obtain a comprehensive and accurate understanding of multi-ethnic populations in Xinjiang area, this study was using a big data analysis of nearly 10 million people aged 18-years-old and above, with Harmonized ATPIII diagnosis standard, to show the prevalence and contributing risk factors of MetS and to provide evidence for preventive interventions against MetS and related diseases in China.

\section{Materials and Methods Study Design and Population}

The study included data from 2019 health-checking program in Xinjiang, the largest autonomous multi-ethnic region in China. All residents of Xinjiang were offered this free checking organized and funded by the government. It contained socioeconomic variables, status of medical resource utilization, consultation and national health screening results, and status of clinic. This study analyzed $57.4 \%$ of the whole Xinjiang population aged $\geq 18$-year s-old. A total of $16,979,107$ residents participated in this health-checking program by 2019 . After excluding individuals who were aged $<18$ years $(n=6,333,609)$, those who were unwilling to participate $(n=457,108)$, those whose data did not contain MetS contributing factors (WC, BP, FBG, TG or HDL $)(n=245,755)$, pregnant women $(\mathrm{n}=96,923)$, potential duplicates $(\mathrm{n}=$ $25,638)$ and unreasonable outliers $(n=74,362)$, a total of $9,745,640$ subjects $(52.7 \%$ female $=5,134,110)$ were finally included in this study (see Figure 1).

\section{Data Collection and Measurement}

At multiple health check sites, uniformly trained staff collected data in accordance with the same standards.

Demographic data and lifestyle risk factors, such as age, sex, marital status, education, smoking status, alcohol consumption, physical activity, were all collected through face-to-face interviews. Waist circumference was measured to 1 decimal point $(0.1 \mathrm{~cm})$, at the middle point between the lowest rib and the highest iliac crest line under exhaled condition, using a measuring tape. Body mass index (BMI) was calculated by dividing weight $(\mathrm{kg})$ by squared height $(\mathrm{m})$. Blood pressure was measured on left arm three times using an automated electronic device (OMRON Model HEM-7071, Omron Company) 


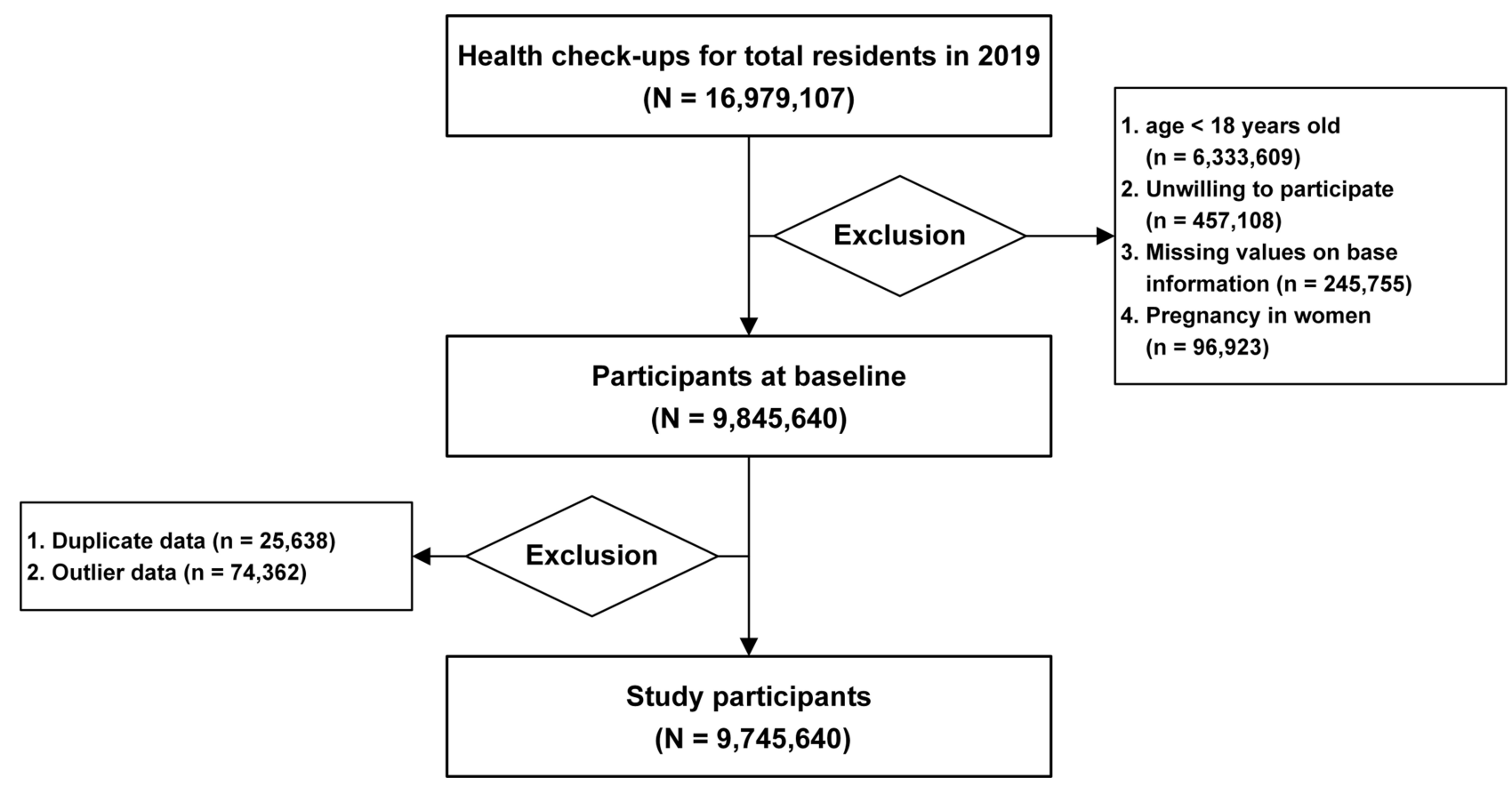

Figure I Flow chart of the study.

after sitting down for 5 min to calculate mean value of the 2nd and 3rd blood pressure readings as the final blood pressure value.

Blood was collected from the antecubital vein after at least $8 \mathrm{~h}$ of fasting, refrigerated, and analyzed within $4 \mathrm{~h}$ at qualified laboratories. TG, HDL-C, LDL-C and FBG were measured according to the equipment and manufacturer's instruction.

\section{Definition of MetS}

In the present study, we used the Harmonized criteria from the National Cholesterol Educational Program (the revised NCEP-ATP III for Asians in 2009 ${ }^{26}$ ) to define MetS, which required the presence of three or more of the following five MetS components: 1) Central obesity (according to the Asian crowd standard, waist circumference $(\mathrm{WC}) \geq 90 \mathrm{~cm}$ in men and $\geq 80 \mathrm{~cm}$ in women); 2) Raised serum triglycerides (high-TG) $\geq 1.7$ $\mathrm{mmol} / \mathrm{L}(150 \mathrm{mg} / \mathrm{dL})$; 3) Low high-density lipoprotein cholesterol (low-HDL-C) $<1.0 \mathrm{mmol} / \mathrm{L}(40 \mathrm{mg} / \mathrm{dL})$ in males and $<1.3 \mathrm{mmol} / \mathrm{L}(50 \mathrm{mg} / \mathrm{dL})$ in females; 4) High blood pressure (high-BP) as $\mathrm{SBP} \geq 130 \mathrm{mmHg}$ or DBP $\geq 85 \mathrm{mmHg}$ or taking antihypertensive drugs; and 5) Raised fasting blood glucose (hyperglycemia, high$\mathrm{FBG}) \geq 5.6 \mathrm{mmol} / \mathrm{L}(100 \mathrm{mg} / \mathrm{dL})$ or taking antihyperglycemia drugs.

\section{Statistical Analysis}

All data analyses were conducted using The R Foundation for Statistical Computing Platform $\mathrm{R}$ version 4.0.2. Variables were summarized as mean for continuous data and percentage or proportion for categorical data. The 95\% CIs were calculated for means, percentages, or proportions. The Student $t$ test, one-way ANOVA and RaoScott $\chi^{2}$ test were used to assess the differences across groups for continuous and categorical variables respectively. A multivariable logistic regression analysis was conducted to evaluate the associated factor for MetS and its components. All tests were two tailed, and $P<0.05$ was considered statistically significant. Outliers are judged by Mahalanobis-MCD (Mahalanobis-Minimum Covariance Determinant) distance, as whit of MCD75 and a chisquare at $P=0.001$ for outliers. ${ }^{27}$

\section{Ethical Approval and Consent to Participate}

The Medical Ethics Committee of the First Affiliated Hospital of Xinjiang Medical University approved the study protocol (20190225-105). The national health examination of whole population in Xinjiang was a health benefit program offered to the residents, and the program mainly covered the common physical examination items as reported in this manuscript. The data were used for 
baseline analysis (such as MetS prevalence and risk factors analysis, etc.) and disease management measures. Collection of the data has been approved by the Autonomous Regional Health Commission, with written Consent Form approved by each and every related resident involved in this research. The use of the national health checking data has been approved by the Primary Health Department, who is responsible for the health information security in China. The content of this article has been examined and approved for scientific publication by the Department. The data are not available without approval.

\section{Results \\ Data from Physical Exams and Laboratory Tests}

General population characteristics are shown in Table 1 and anthropometry measurements, blood test results and health behavior characteristics are shown in Table 2 with the averages of BMI, WC, SBP, DBP, LDL-c, HDL-c, TC, FBG and the percentage of smoking, drinking and physical activity. The results showed that all averages were significantly

Table I Sample Descriptive Characteristic $(N=9,745,640)$

\begin{tabular}{|c|l|l|}
\hline Variables & $\begin{array}{l}\text { Total } \\
(\mathbf{n}=9,745,640)\end{array}$ & $\begin{array}{l}\text { Percentage } \\
\text { (\%) }\end{array}$ \\
\hline $\begin{array}{l}\text { Sex } \\
\text { Male }\end{array}$ & $5,134,110$ & 52.68 \\
\hline Ages & & \\
I8-39 ys & $4,412,117$ & 45.27 \\
$40-59$ ys & $3,731,961$ & 38.29 \\
$\geq 60$ ys & $1,601,562$ & 16.43 \\
\hline Ethnicity & & \\
Han & $2,481,787$ & 25.47 \\
Uyghur & $5,645,419$ & 57.93 \\
Kazak & 799,942 & 8.21 \\
Hui & 404,253 & 4.15 \\
Kyrgyz & 113,716 & 1.17 \\
Mongolian & 79,165 & 0.81 \\
Other & 221,358 & 2.27 \\
\hline Household & & \\
registration & & \\
Rural & $6,870,013$ & 70.49 \\
\hline Geographical location & & 58.79 \\
\hline North & $4,364,234$ & \\
\hline Education & & \\
6years or less & $5,729,947$ & \\
\hline
\end{tabular}

different among different groups of sex, age, ethnicity, household registration, geographical location and education $(P<0.0001)$.

In terms of sex, except that the HDL-c in male group was lower than that in female, the other indexes of male group were higher than that in female $(P<0.0001)$. Smoking and drinking accounted for the highest level in the 40-59 age group, and physical activity was the highest in 60-years and over group.

In terms of age, all indexes were the lowest in 18- to 39-year-old group; and the highest weight, BMI and TG were observed in 60-years and over group with middle ranges for 40 - to 59-year group $(P<0.0001)$; the highest drinking and smoking rates were in the 40- to 59-years group while the highest physical activities in 60-years and over group $(P<0.0001)$.

In terms of ethnicity, in Uyghur, TC was the lowest and all other indexes were in middle or lower levels; in Han, TC was the highest, and other indexes were at higher levels; in Kazak, SBP and HDL-c were the highest, TG was the lowest, and other indexes were at higher levels; in Hui, FBG was the highest, and other indexes were at higher levels; in Kyrgyz, LDL-c was the highest, and weight, WC, SBP, and DBP were the lowest, and other indexes were at lower levels; in Mongolian, height, weight, BMI, WC, DBP, and HDL-c were the highest and other indexes were at lower levels $(P<0.0001)$. As for lifestyles, Kyrgyz had the highest drinking rates followed by Mongolian, Kazak, Uyghur, Han, and Hui; Kyrgyz also had the least people doing physical activity followed by Uyghur, Kazak, Hui, Mongolian, and Han.

\section{Prevalence of Metabolic Syndrome}

The prevalence of MetS was markedly different between sex, household registration, residential geographical location and education, showing higher in male $(21.60 \%)$, in 60 -years and over age group (39.22\%), in city and town residents $(23.03 \%)$, in North Xinjiang area (24.78\%), and in 7 or more-years education group $(24.54 \%)$. There were significant differences in ages. Prevalence was $9.56 \%$ in 18 - to 39 -year group and increased up to $39.22 \%$ in $\geq 60$ years group. There were significant differences in prevalence between ethnicities, showing the highest in Hui (28.19\%), followed by Han $(27.3 \%)$, others $(18.61 \%)$, Uyghur (18.56\%), Mongolian (17.98\%), Kazak (17.87\%) and Kyrgyz (14.44\%) (Table 3).

The prevalence of five MetS components showed that central obesity was the highest $(51.71 \%)$, followed by 


\begin{tabular}{|c|c|c|c|c|c|c|c|c|c|c|c|c|c|c|}
\hline 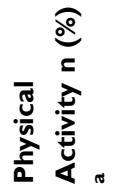 & 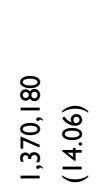 & & 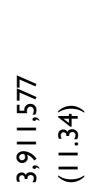 & 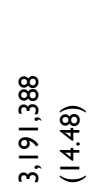 & 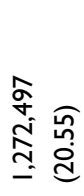 & & 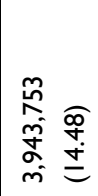 & 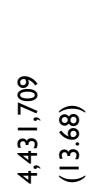 & & 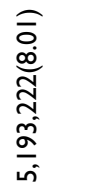 & 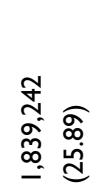 & 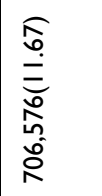 & 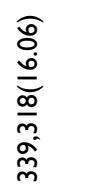 & 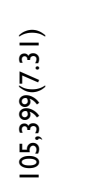 \\
\hline 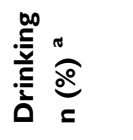 & $\begin{array}{l}\frac{\infty}{o} \stackrel{\widehat{o}}{\stackrel{p}{p}} \\
\stackrel{\stackrel{m}{=}}{=}\end{array}$ & & 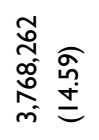 & 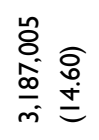 & 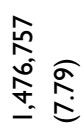 & & 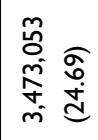 & 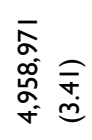 & & 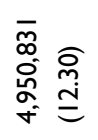 & 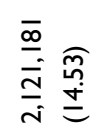 & 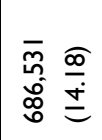 & 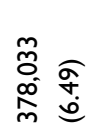 & 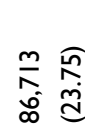 \\
\hline 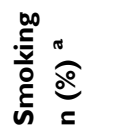 & 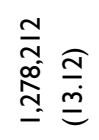 & & 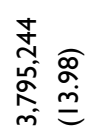 & 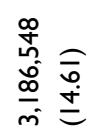 & 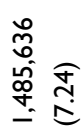 & & 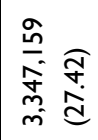 & 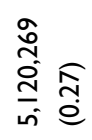 & & 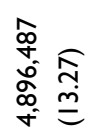 & 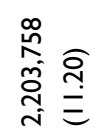 & 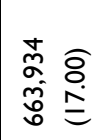 & 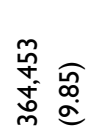 & 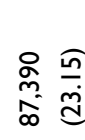 \\
\hline О. & 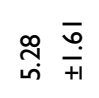 & & $\begin{array}{c}\infty \\
\stackrel{\alpha}{\sigma} \\
\stackrel{+}{+} \\
+1\end{array}$ & 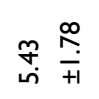 & $\stackrel{\infty}{\stackrel{\infty}{n}} \stackrel{\frac{n}{i}}{i}$ & & 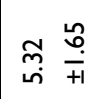 & 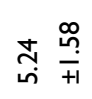 & & 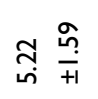 & 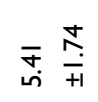 & 공 & 용 & $\bar{\sim} \underset{i}{\stackrel{Y}{+1}}$ \\
\hline 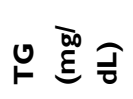 & $\stackrel{m}{\stackrel{9}{=}}$ & & 京 & 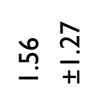 & 뜬 $\frac{8}{+1}$ & & $\stackrel{\text { ñ }}{\underline{\underline{m}}} \frac{m}{+1}$ & m & & 部 & $\stackrel{\infty}{\stackrel{\infty}{\dddot{m}}} \frac{\tilde{m}}{++1}$ & $\stackrel{ \pm}{ \pm} \frac{8}{+1}$ & $\stackrel{\text { 늠 }}{\underline{+1}}$ & 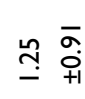 \\
\hline 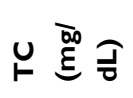 & 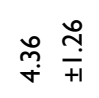 & & 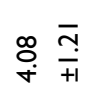 & 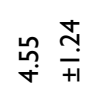 & $\underset{+}{\sigma} \frac{m}{+1}$ & & 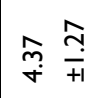 & 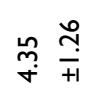 & & $\underset{+}{\mathbb{*}} \frac{\underset{+1}{+1}}{1}$ & $\stackrel{\infty}{\stackrel{\infty}{\rightarrow}} \frac{\stackrel{t}{m}}{++1}$ & 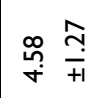 & $\underset{+}{\frac{7}{+}} \frac{\sigma}{+1}$ & $\stackrel{\overbrace{}}{\stackrel{\sim}{~}} \frac{\widehat{s}}{+1}$ \\
\hline 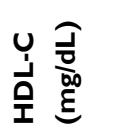 & 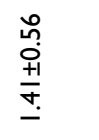 & & 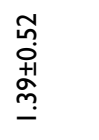 & 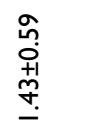 & 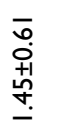 & & 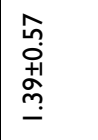 & 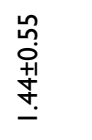 & & 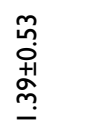 & $\begin{array}{l}\stackrel{8}{0} \\
\text { +1 } \\
\text { +1 } \\
\stackrel{+}{+}\end{array}$ & $\begin{array}{l}\frac{2}{f} \\
0 \\
+1 \\
+1 \\
\underline{f}\end{array}$ & 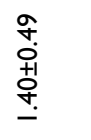 & 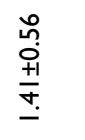 \\
\hline & 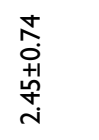 & & 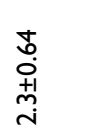 & $\begin{array}{l}\stackrel{2}{0} \\
\text { +1 } \\
\hat{n} \\
\text { ñ }\end{array}$ & 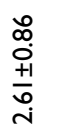 & & 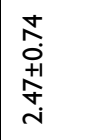 & 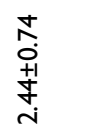 & & 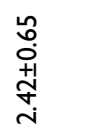 & 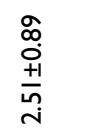 & 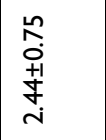 & 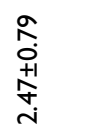 & 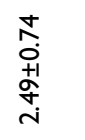 \\
\hline 总 & 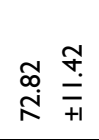 & & 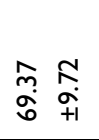 & 突 & $\underset{\frac{\sigma}{i}}{\frac{a}{i}}$ & & $\underset{\dot{i}}{\stackrel{\stackrel{n}{m}}{\overline{+1}}}$ & 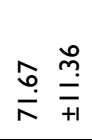 & & 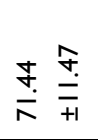 & 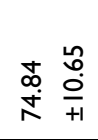 & 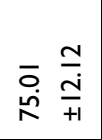 & 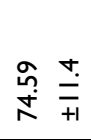 & $\stackrel{\infty}{\stackrel{\infty}{x}} \stackrel{\stackrel{f}{+}}{\frac{1}{+1}}$ \\
\hline 全 & 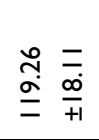 & & 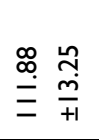 & 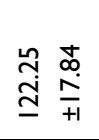 & 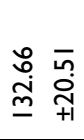 & & 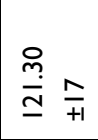 & 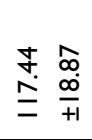 & & 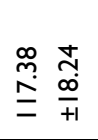 & 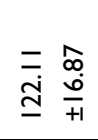 & 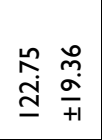 & 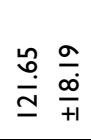 & 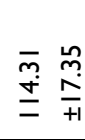 \\
\hline उ है & 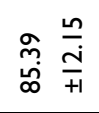 & & 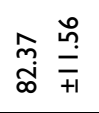 & 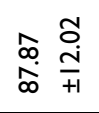 & $\underset{\infty}{\stackrel{\frac{n}{n}}{\frac{1}{4}}}$ & & 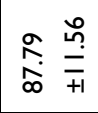 & $\underset{\infty}{\underset{\infty}{\sim}} \underset{+1}{\stackrel{\sim}{+}}$ & & $\underset{\infty}{\infty} \stackrel{\substack{n \\
\infty}}{\stackrel{n}{+1}}$ & 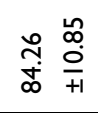 & 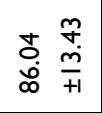 & 可 & 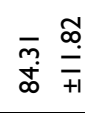 \\
\hline 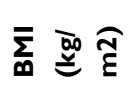 & $\begin{array}{ll}\bar{\infty} & 0 \\
\dot{\sim} & \\
\dot{+} & +\end{array}$ & & 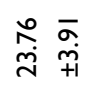 & 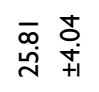 & 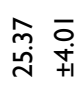 & & 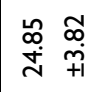 & 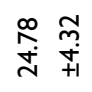 & & $\begin{array}{ll}\stackrel{2}{*} \\
\stackrel{+}{+} \\
\stackrel{+}{+}\end{array}$ & 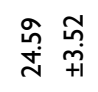 & 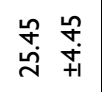 & 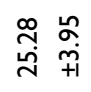 & 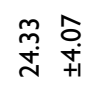 \\
\hline 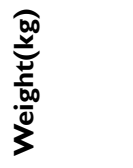 & $\begin{array}{l}\frac{\hat{2}}{\mathrm{i}} \\
\frac{+1}{+} \\
\frac{\dot{0}}{0}\end{array}$ & & 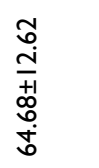 & 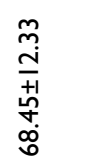 & 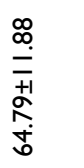 & & 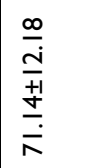 & 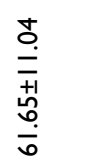 & & 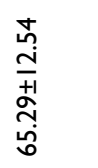 & 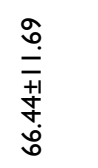 & 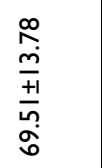 & 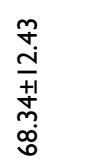 & 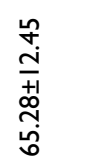 \\
\hline 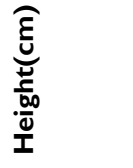 & $\begin{array}{l}\alpha \\
\alpha \\
o \\
+1 \\
\underline{0} \\
\underline{3}\end{array}$ & & 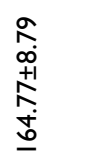 & 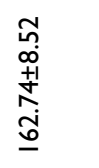 & $\begin{array}{l}\bar{a} \\
\frac{+1}{\hat{+1}} \\
\text { مَ }\end{array}$ & & $\begin{array}{l}\stackrel{n}{o} \\
+1 \\
+1 \\
\frac{0}{0} \\
\underline{0}\end{array}$ & $\begin{array}{l}\hat{N} \\
0 \\
+1 \\
\infty \\
0 \\
\underline{n}\end{array}$ & & 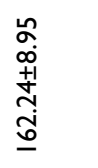 & 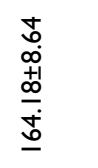 & $\begin{array}{l}m \\
\alpha \\
\infty \\
+1 \\
m \\
\\
\underline{0}\end{array}$ & 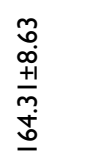 & 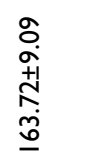 \\
\hline 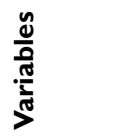 & $\begin{array}{l}\overline{0} \\
\text { ठँग }\end{array}$ & 品 & $\begin{array}{l}\text { an } \\
\hat{0} \\
\underline{\omega} \\
\underline{\omega}\end{array}$ & $\begin{array}{l}\text { à } \\
\text { مै } \\
\text { के }\end{array}$ & 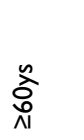 & ñ & $\sum^{\bar{\omega}}$ & 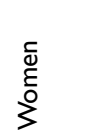 & 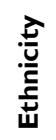 & 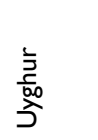 & $\underset{\text { I }}{\text { I }}$ & 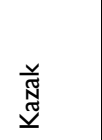 & 言 & $\begin{array}{l}\hat{N} \\
\text { 离 } \\
\underline{\underline{y}}\end{array}$ \\
\hline
\end{tabular}




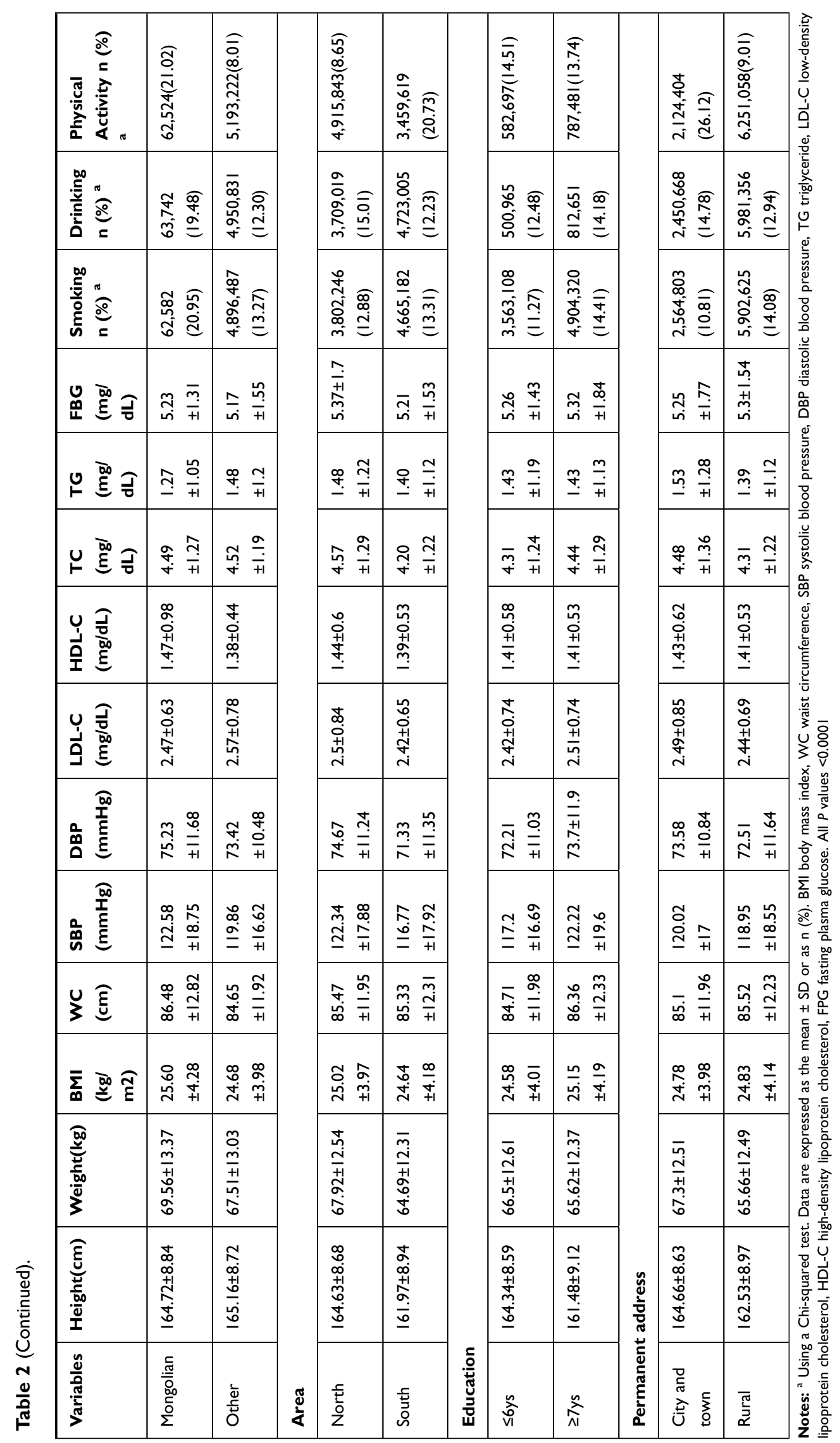


Table 3 Rates of Metabolic Syndrome and Its Components $(\mathrm{N}=9,745,640)$

\begin{tabular}{|c|c|c|c|c|c|c|c|c|c|c|c|c|}
\hline \multirow{3}{*}{ Variables } & \multirow{2}{*}{\multicolumn{2}{|c|}{$\begin{array}{l}\text { Metabolic } \\
\text { syndrome }\end{array}$}} & \multicolumn{10}{|c|}{ MetS Component } \\
\hline & & & \multicolumn{2}{|c|}{ Central obesity } & \multicolumn{2}{|c|}{$\begin{array}{l}\text { High } \\
\text { triglycerides }\end{array}$} & \multicolumn{2}{|c|}{$\begin{array}{l}\text { Low high- } \\
\text { density } \\
\text { lipoprotein } \\
\text { cholesterol }\end{array}$} & \multicolumn{2}{|c|}{$\begin{array}{l}\text { Elevated blood } \\
\text { pressure }\end{array}$} & \multicolumn{2}{|c|}{$\begin{array}{l}\text { Elevated } \\
\text { fasting plasma } \\
\text { glucose }\end{array}$} \\
\hline & $\%$ & $P$ & $\%$ & $P$ & $\%$ & $P$ & $\%$ & $P$ & $\%$ & $P$ & $\%$ & $P$ \\
\hline Total & 20.85 & $<0.001$ & 51.71 & $<0.001$ & 23.71 & $<0.001$ & 4.68 & $<0.001$ & 23.16 & $<0.001$ & 26.59 & $<0.001$ \\
\hline \multicolumn{13}{|l|}{ Sex } \\
\hline Female & 21.56 & \multirow{2}{*}{$<0.001$} & 60.83 & \multirow{2}{*}{$<0.001$} & 19.37 & \multirow{2}{*}{$<0.001$} & 5.69 & \multirow{2}{*}{$<0.001$} & 21.70 & \multirow{2}{*}{$<0.001$} & 28.27 & \multirow{2}{*}{$<0.001$} \\
\hline Male & 20.06 & & 41.56 & & 28.53 & & 9.89 & & 24.81 & & 25.80 & \\
\hline \multicolumn{13}{|l|}{ Ages } \\
\hline 18-39 & 9.56 & \multirow{3}{*}{$<0.001$} & 40.79 & \multirow{3}{*}{$<0.001$} & 18.66 & \multirow{3}{*}{$<0.001$} & 7.46 & \multirow{3}{*}{$<0.001$} & 6.71 & \multirow{3}{*}{$<0.001$} & 16.94 & \multirow{3}{*}{$<0.001$} \\
\hline $40-59$ & 26.32 & & 60.86 & & 28.15 & & 7.30 & & 28.12 & & 30.48 & \\
\hline$\geq 60$ 岁 & 39.22 & & 60.48 & & 27.24 & & 9.15 & & 56.96 & & 44.14 & \\
\hline \multicolumn{13}{|l|}{ Ethnicity } \\
\hline Uyghur & 18.56 & \multirow{7}{*}{$<0.001$} & 52.80 & & 22.98 & & 7.88 & & 20.69 & & 23.89 & \\
\hline Han & 26.39 & & 48.93 & & 27.75 & & 8.11 & & 26.49 & & 32.69 & \\
\hline Kazak & 17.98 & & 52.47 & $<0001$ & 16.78 & $<0001$ & 4.53 & $<0001$ & 26.96 & $<0001$ & 23.84 & $<0001$ \\
\hline Hui & 28.19 & & 54.85 & $<0.001$ & 26.04 & $<0.001$ & 8.09 & $<0.001$ & 26.21 & $<0.001$ & 35.68 & $<0.001$ \\
\hline Kyrgyz & 14.44 & & 47.01 & & 16.69 & & 7.79 & & 19.55 & & 25.57 & \\
\hline Mongolian & 17.87 & & 55.94 & & 17.74 & & 3.53 & & 27.18 & & 24.17 & \\
\hline Other & 18.61 & & 47.51 & & 23.45 & & 9.66 & & 30.13 & & 21.69 & \\
\hline Permanent a & dress & & & & & & & & & & & \\
\hline City and & 23.03 & $<0001$ & 51.12 & $<0001$ & 26.65 & $<0001$ & 7.94 & $<0001$ & 23.81 & $<0001$ & 26.56 & 122 \\
\hline Rural & 19.94 & -0.001 & 51.96 & -0.001 & 22.48 & -0.001 & 7.57 & -0.001 & 22.90 & -0.001 & 26.61 & $0.12<$ \\
\hline Area & & & & & & & & & & & & \\
\hline North & 24.78 & $<0001$ & 52.18 & $<0001$ & 24.91 & $<0 \Omega 01$ & 7.18 & $<0001$ & 27.20 & $<0001$ & 30.28 & $<0001$ \\
\hline South & 17.66 & 0.001 & 51.33 & -0.001 & 22.73 & -0.001 & 8.08 & 10.001 & 19.90 & - & 23.60 & -0.001 \\
\hline Education & & & & & & & & & & & & \\
\hline 6 years or & 18.26 & $<0001$ & 48.92 & $<0001$ & 23.51 & $<0001$ & 7.34 & $<0001$ & 17.49 & $<0001$ & 24.89 & $<0001$ \\
\hline 7 years or & 24.54 & -0.001 & 55.70 & -0.001 & 23.98 & -0.001 & 8.16 & -0.001 & 31.27 & 10.0 & 29.01 & -0.001 \\
\hline Smoking & & & & & & & & & & & & \\
\hline Yes & 20.45 & $<0001$ & 44.24 & $<0001$ & 32.00 & $<0001$ & 8.02 & $<0001$ & 21.25 & $<0001$ & 27.90 & $<0001$ \\
\hline No & 20.94 & & 52.84 & & 22.45 & 10.001 & 7.62 & 10.001 & 23.46 & 10.001 & 26.39 & -0.001 \\
\hline Drinking & & & & & & & & & & & & \\
\hline Yes & 20.85 & 0.978 & 46.09 & $<0001$ & 30.35 & $<0001$ & 9.01 & $<0001$ & 23.37 & $<0001$ & 26.50 & 0009 \\
\hline No & 20.85 & 0.010 & 52.59 & & 22.67 & & 7.47 & & 23.14 & 10.001 & 26.61 & 0.000 \\
\hline Physical & & & & & & & & & & & & \\
\hline Yes & 25.80 & $<0001$ & 52.85 & $<0001$ & 27.11 & $<0001$ & 8.96 & $<0001$ & 31.46 & $<0001$ & 28.64 & $<0001$ \\
\hline No & 20.04 & -0.001 & 51.53 & -0.001 & 23.15 & -0.001 & 7.47 & -0.001 & 21.81 & -0.001 & 26.26 & -0.001 \\
\hline
\end{tabular}

high-FBG (26.59\%), high-TG (23.71), high-BP (23.16\%), and low-HDL-C (4.68\%). Central obesity and high-FBG were more prevalent in women than that in men; high-TG, low-HDL-C, high-BP were more often in men than that in women. As to age, the prevalence of high-FBG, high-BP and low-HDL-C were the highest in the 60-years and over age group, while central obesity and high-TG were the highest in 40- to 59-years-old. The prevalence of highFBG, high-TG and low-HDL-C were remarkably higher in Hui $(35.68 \%, 26.04 \%$ and $8.09 \%, P<0.0001)$, and Han 


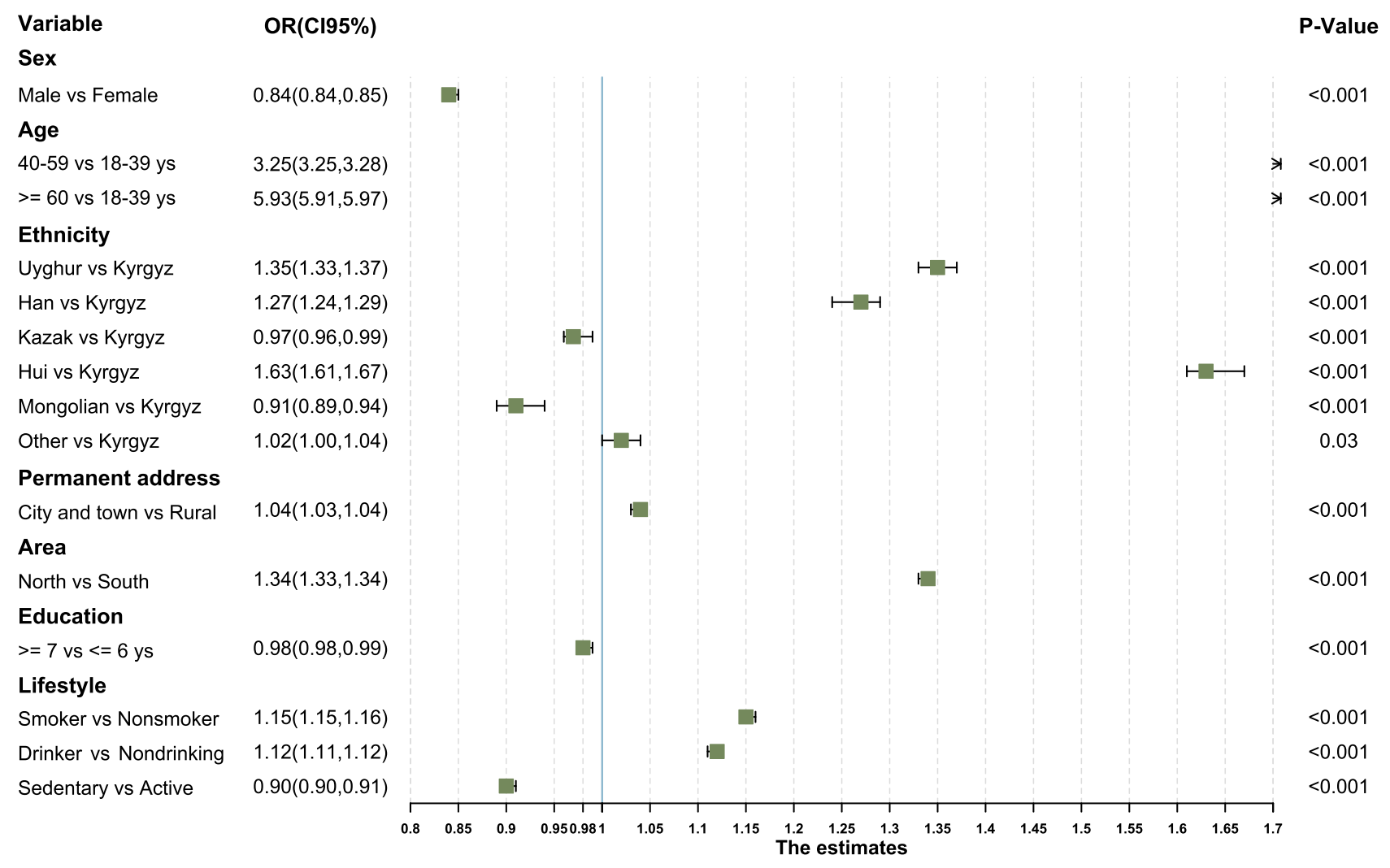

Figure 2 Odds ratios and $95 \% \mathrm{Cls}$ for the associations of MetS with population and health behavior characteristics. The arrows in the figure indicate that the range of corresponding values exceed the plotting area.

$(32.69 \%, 27.75 \%$ and $8.11 \%, P<0.0001)$ than that in other ethnic groups. The prevalence of central obesity and highBP of Mongolian were significantly higher $(55.94 \%$ and $27.18 \%, P<0.0001)$ than that in other ethnic groups.

The prevalence of MetS and its components were higher in smoking and drinking groups except for central obesity and high-BP. The prevalence of MetS and its components increased in participants with higher physical activity levels (Table 3 ).

\section{Influential Factors Associated with MetS and Its Components}

The logistic regression analysis was performed to identify factors affecting MetS and its components in Figures 2-7. The risk to have MetS was $1.19(1 / 0.84)$ times less in women relative to that in men $(95 \%$ confidence interval [CI] 0.84-0.85, $P<0.0001)$. The risk was 3.25 and 5.93 times higher in 40- to 59-year group and $\geq 60$-years group than that in 18-39-year group (95\% CI: 3.25-3.38, 5.91-5.97, $P<0.0001$ ). The risk was $1.35,1.27,1.63$ times higher in Uyghur, Han, Hui than that in Kyrgyz (95\% CI: 1.33-1.37,
$1.24-1.29,1.61-1.67, P<0.001)$; it was $1 / 0.97,1 / 0.91$ time lower than that in Kazak, Mongolian (95\% CI: 0.96-0.99, 0.89-0.94). The risk was 1.04 times higher in cities and towns than that in rural subjects $(95 \% \mathrm{CI}$ : $1.03-1.04, P<0.001)$, and 1.34 times higher in north Xinjiang residents than that in south residents $(95 \% \mathrm{CI}$ : $1.33-1.34, P<0.001)$. The risk was $1 / 0.98$ times lower in $\geq 7$-year education group than that in 6 years and less education subjects (95\% CI: $0.98-0.99, P<0.001)$. The risk was $1.15,1.12$ times higher in smoking and drinking groups than that in non-smoking and non-drinking groups $(95 \%$ CI: $1.15-1.16, \quad 1.11-1.12, \quad P<0.001)$ (Figure 2).

The risk of central obesity was 2.38 (1/0.42) times higher in women relative to that in men (95\% CI: $0.42-0.42$, $P<0.001)$. Among ethnic groups, Uyghur and Mongolian had higher risk to have central obesity [OR (95\% CI): 1.27 (1.25-1.29), 1.16 (1.14, 1.18)], while Han had less risk than others [OR (95\% CI): $0.79(0.79-0.81)]$. The risk of central obesity was 1/0.98 times lower in 7 year or above education group (95\% CI: $0.98-0.99, P<0.001)$. Living in cities and towns, Northerners, smokers and drinkers were more at risk of central obesity (Figure 3). 


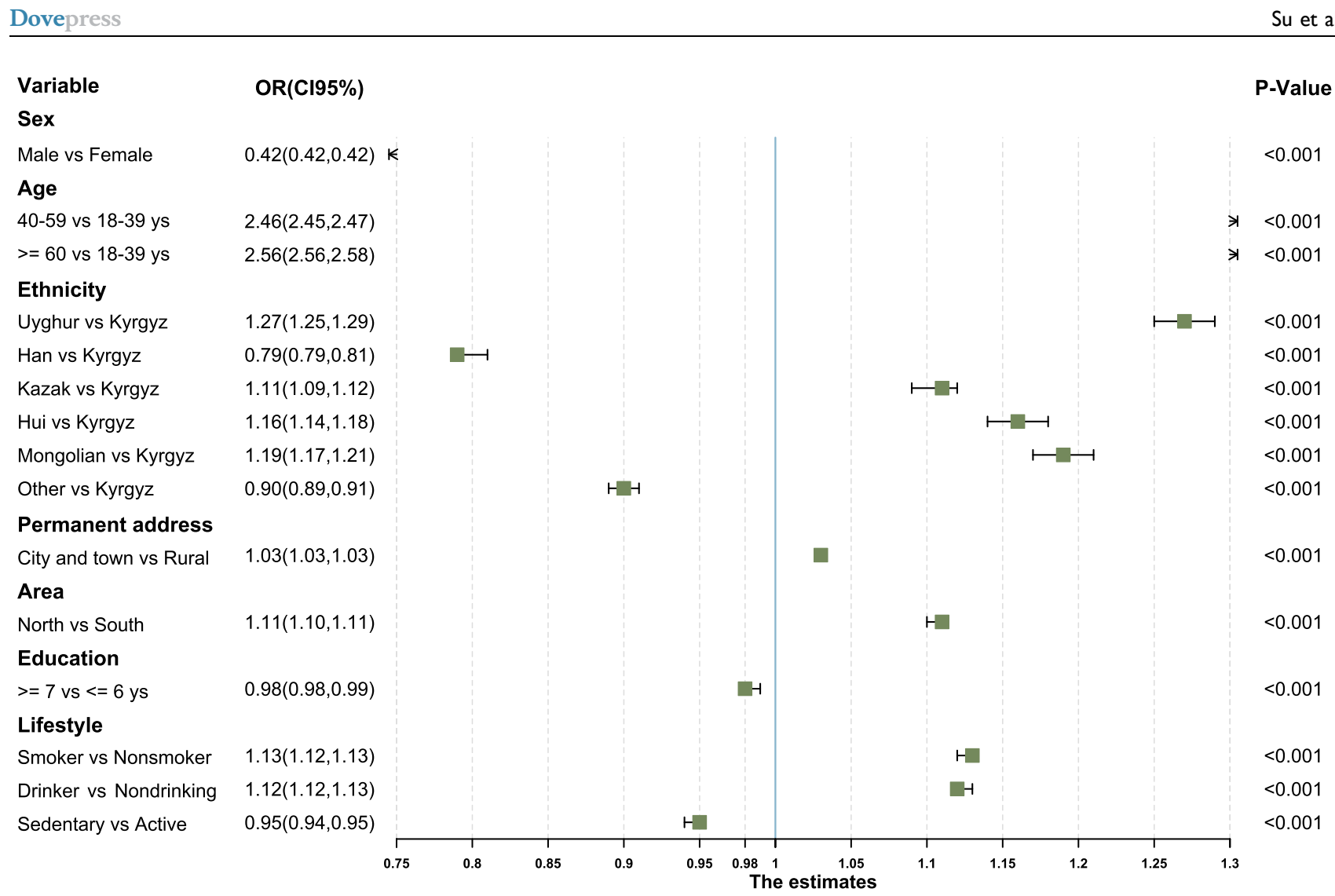

Figure 3 Odds ratios and $95 \% \mathrm{Cls}$ for the associations of central obesity with population and health behavior characteristics.

The risk of high-TG levels was 1.54 times higher in men than that in women $(95 \% \mathrm{CI}: 1.53-1.54, P<0.001)$. Among ethnic groups, Hui and Han had higher risk to have high-TG than other ethnic groups [OR $(95 \%$ CI):1.67 (1.64-1.70), $1.62(1.58,1.64)]$, while Mongolian and Kazak had lower risk than others. The risk of high-TG was $1 / 0.94$ times lower in $\geq 7$-year education group $(95 \%$ CI: $0.94-0.94, P<0.001)$. Living in cities and town, Northerners, smokers and drinkers were more at risk of high-TG (Figure 4).

The risk of low-HDL-C was 1.93 times higher in men than that in women $(95 \% \mathrm{CI}: 1.92-1.94, P<0.001)$. Among ethnic groups, Hui and Han had higher risk to have low-HDL-C than other ethnic groups [OR $(95 \%$ CI): 1.11 (1.08-1.14), 1.07 (1.05-1.10)], while Mongolian and Kazak had remarkably lower risk than others. The risk of low-HDL-C was 1.09 times higher in $\geq 7$ year education group (95\% CI: $1.08-1.10, P<0.001$ ). Living in cities and towns, drinkers had higher and Northerners, smokers had lower risk to have low-HDL-C (Figure 5).

The risk of high-FBG was 1.15 times higher in men than that in women $(95 \% \mathrm{CI}: 1.14-1.15, P<0.001)$.
Among ethnic groups, Hui and Han had higher risk to high-FBG than other ethnic groups [OR $(95 \% \mathrm{CI})]$. The risk of high-FBG was 1.25 (1.23-1.26), 1.04 (1.02-1.05), while Mongolian and Kazak had lower risk than others (Figure 6).

The risk of high-BP was 1.23 times higher in men than that in women $(95 \% \mathrm{CI}: 1.23-1.24, P<0.001)$. Among ethnic groups, Kazak and Mongolian had more risk to high-BP than other ethnic groups [OR (95\% CI): 1.28 (1.26-1.31), 1.15 (1.12-1.17)], while Han and Hui had lower risk than others. The risk of high-BP was 1.19 times higher in $\geq 7$-year education group than that in 6 year and less education subjects (95\% CI: $1.18-1.19$, $P<0.001)$. Living in cities and towns, smokers have less and Northerners, drinkers had more risk to high-BP (Figure 7).

\section{Discussion}

This study was carried out in Xinjiang, a typical multiethnic province in the world. Xinjiang is the largest province in China, with an area of 1.66 million square kilometers, accounting for 1/6 of China's total land area. There are 55 ethnic groups well mixed in Xinjiang, with 


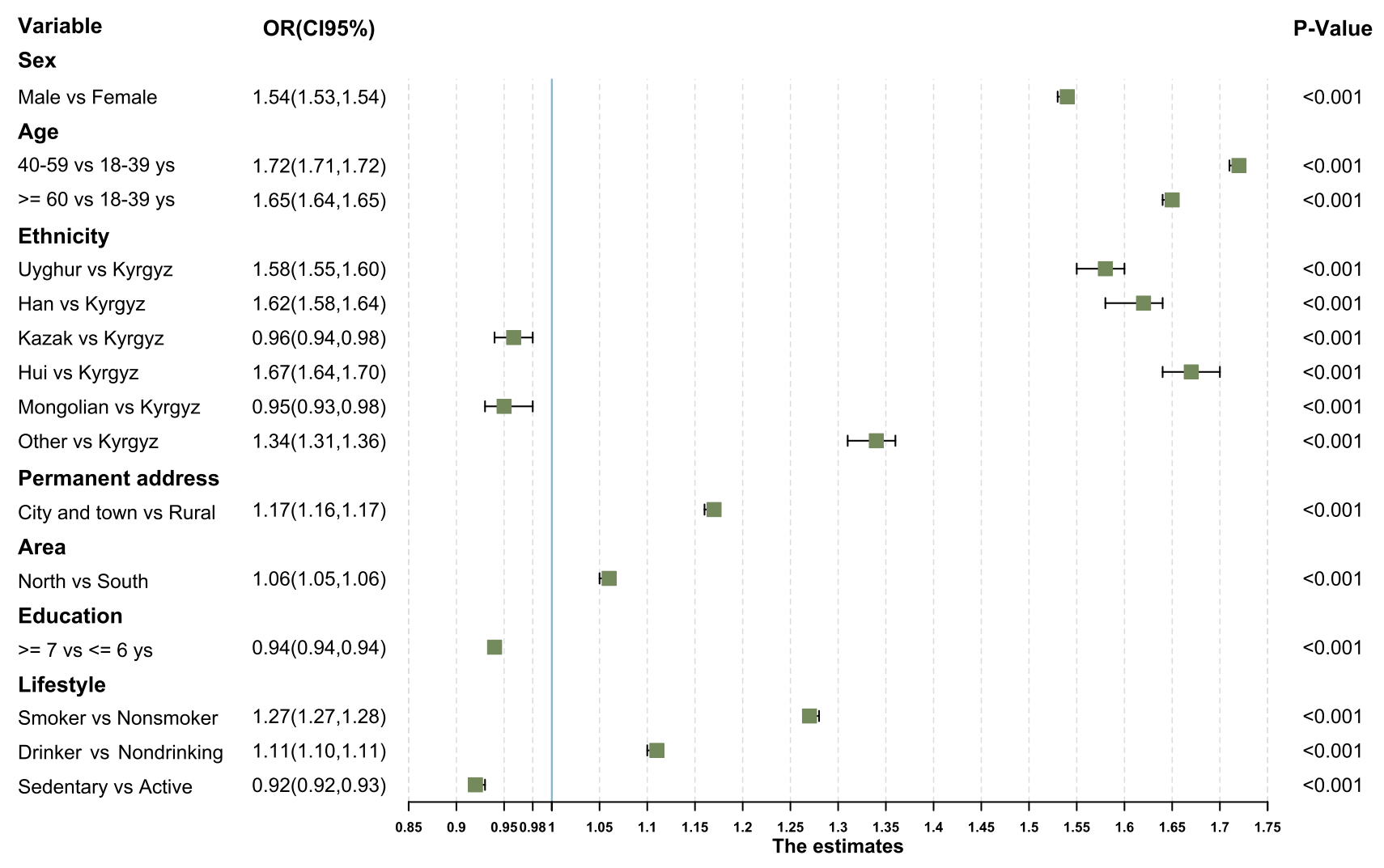

Figure 4 Odds ratios and $95 \% \mathrm{Cls}$ for the associations of high-TG with population and health behavior characteristics. The arrows in the figure indicate that the range of corresponding values exceed the plotting area.

a resident population of 24.8676 million. The top 6 ethnic groups in Xinjiang are Uygur, Han, Kazakh, Hui, Mongolian and Kyrgyz. Xinjiang is divided into Northern and Southern Xinjiang because of Tianshan Mountain in the middle. Due to the geographical location and the topography of the Taklimakan Desert, which is the second largest desert in the world in southern Xinjiang, the climates of north and south Xinjiang are very different with temperate continental semi-arid in Northern and arid climate in Southern Xinjiang respectively. Geographical environment and ethnic origin may affect the occurrence and development of metabolic diseases. Therefore, this study not only analyzed the common demographic characteristics for people to live in, but also performed analysis of six ethnic groups in both Northern and Southern Xinjiang. Although the objects of this study were one comprehensive and multi-ethnic population in Xinjiang, considering the huge number of the sampling size, the analysis of each individual subgroup would still be valid to reflect true statistical outcome.

This study showed the prevalence of MetS in this large comprehensive multi-ethnic population in Xinjiang was $20.85 \%$, which was consistent with that of the world population. $^{28}$ This rate is lower than those of Western countries, United States, ${ }^{6}$ other Asian countries, and Chinese studied on other area of China; but higher than that in rural areas in Xinjiang reported in a study conducted previously in multi-ethnic adults in 2017 $(14.43 \%) .^{12}$ It is worth noting that results of previous studies demonstrated that the prevalence of MetS in monoethnic population was always higher than that in mixed or multi-ethnic population. Reason for such difference may be that prevalence of MetS is always low in certain minority ethnic groups to contribute to the average prevalence of multi-ethnic population. This study demonstrated that Kazakh, Mongolian and Kyrgyz had lower prevalence of MetS.

Interestingly, the results showed the lowest prevalence of MetS in Kyrgyz with the highest smoking and drinking rates and the lowest physical activity rate. Lifestyle here did not play a major role in determining the prevalence of MetS in Kyrgyz. Similar phenomenon was observed in Kazak and Mongolian too. Compared with other ethnic groups, these groups had higher BMI, WC, SBP but lower TC and TG. It is speculated that genetic background of these ethnic groups may play 


\section{Variable}

Sex

Male vs Female

Age

$40-59$ vs $18-39$ ys

$>=60$ vs $18-39$ ys

Ethnicity

Uyghur vs Kyrgyz

Han vs Kyrgyz

Kazak vs Kyrgyz

Hui vs Kyrgyz

Mongolian vs Kyrgyz

Other vs Kyrgyz

Permanent address

City and town vs Rural

Area

North vs South

Education

$>=7 \mathrm{vs}<=6 \mathrm{ys}$

Lifestyle

Smoker vs Nonsmoker

Drinker vs Nondrinking

Sedentary vs Active

OR(C|95\%)

P-Value

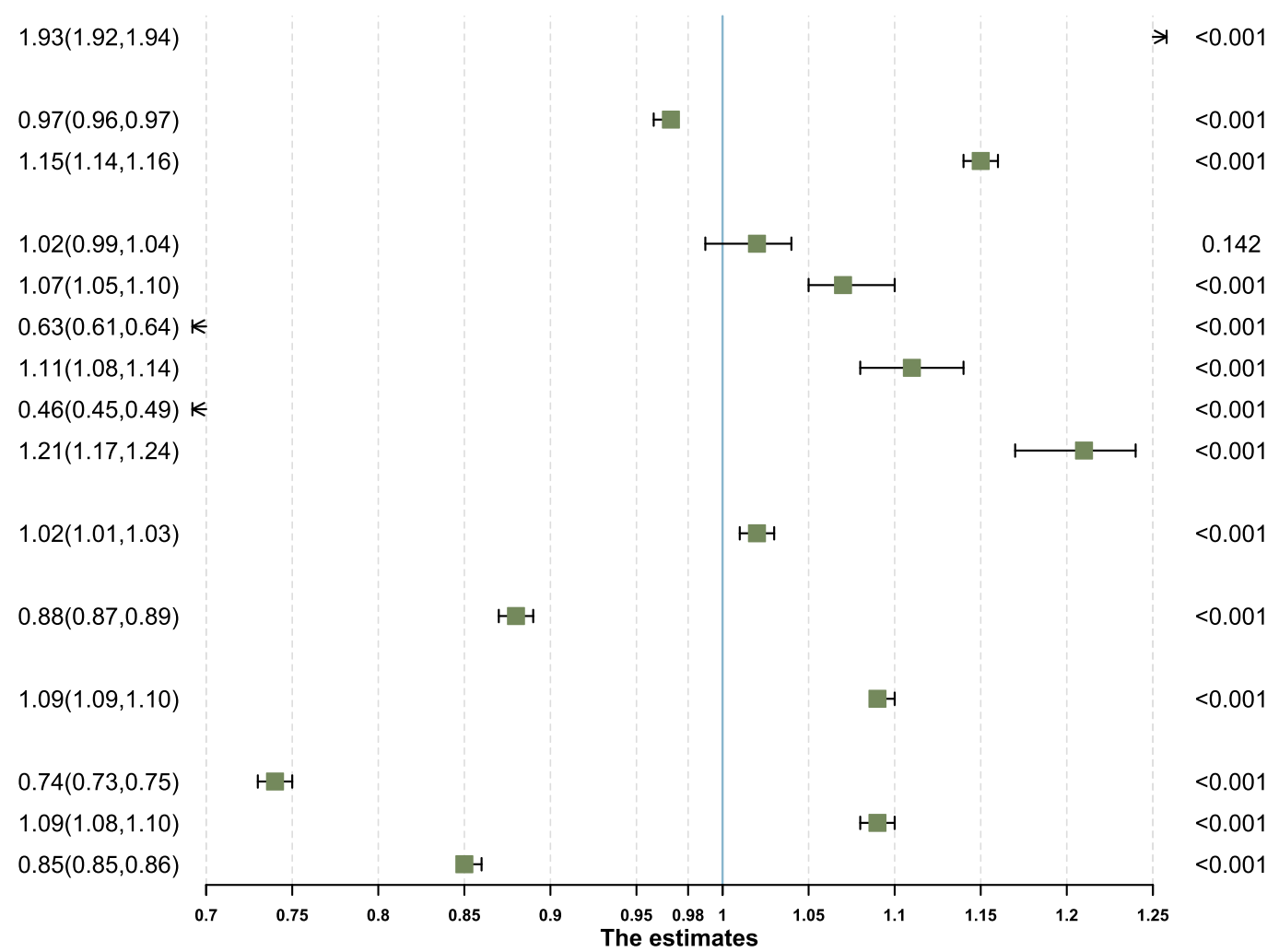

Figure 5 Odds ratios and $95 \% \mathrm{Cls}$ for the associations of low-HDL-C with population and health behavior characteristics. The arrows in the figure indicate that the range of corresponding values exceed the plotting area.

a dominant role in determining prevalence of MetS. In this study, higher HDL-C and less high-TG risk were found in these three ethnic groups, suggesting that hereditary factors might facilitate the production of HDL-C and metabolism of $\mathrm{TG}$ to reduce MetS occurrence. Coincidentally, in 2018, Zhang et $\mathrm{al}^{29}$ identified a rare frame shift variant in the LIMA1 (also known as EPLIN or SREBP3) gene in Kazakh ethnicity with inherited low LDL-C and reduced cholesterol absorption. Several other studies proved that Kazak, Mongolian and Kyrgyz had closer genetic background among them than that in other ethnic groups. ${ }^{30-33}$ Therefore, it is possible that Mongolian and Kyrgyz people may have this unique genetic background too. This is worthy of further study to clarify this issue.

In terms of gender, the prevalence of MetS in women was lower than that in men, but multivariate regression analysis showed that the risk of MetS was higher in women than that in men under adjusting age, ethnic group, region and lifestyle. It is consistent with previous research. ${ }^{12,14,34}$ Postmenopausal status of women increased the prevalence of MetS because of IR and central obesity. ${ }^{35}$
From living in urban or rural area, the prevalence and risk of MetS in urban residents were higher than that in rural residents. With rapid development of urbanization, people's lifestyle and diet are changing too. From the univariate and multivariate analysis of the influencing factors and MetS components in this study, although there were more people doing physical activity in urban area and less smoking population, the rate of MetS was significantly higher in urban than that in rural residents. High-drinking population in urban residents contributed to the high prevalence of MetS as drinking may cause high-TC, low-HDL -C, which promoted MetS. ${ }^{36-38}$ Alcohol restriction should be considered in the health education of urban residents.

Based on the geographical location, the prevalence of MetS in Northern Xinjiang was much higher than that in Southern Xinjiang, and the risk for MetS was also higher in Northern than that in Southern Xinjiang residents. Northerners were more likely to suffer from central obesity, high-TG, high-BP and high-BFG, but had a lower risk of pathological high- and low-density lipoproteins. In fact, residents in Northern Xinjiang ate high-calorie food and drank more alcohol than residents in Southern Xinjiang 


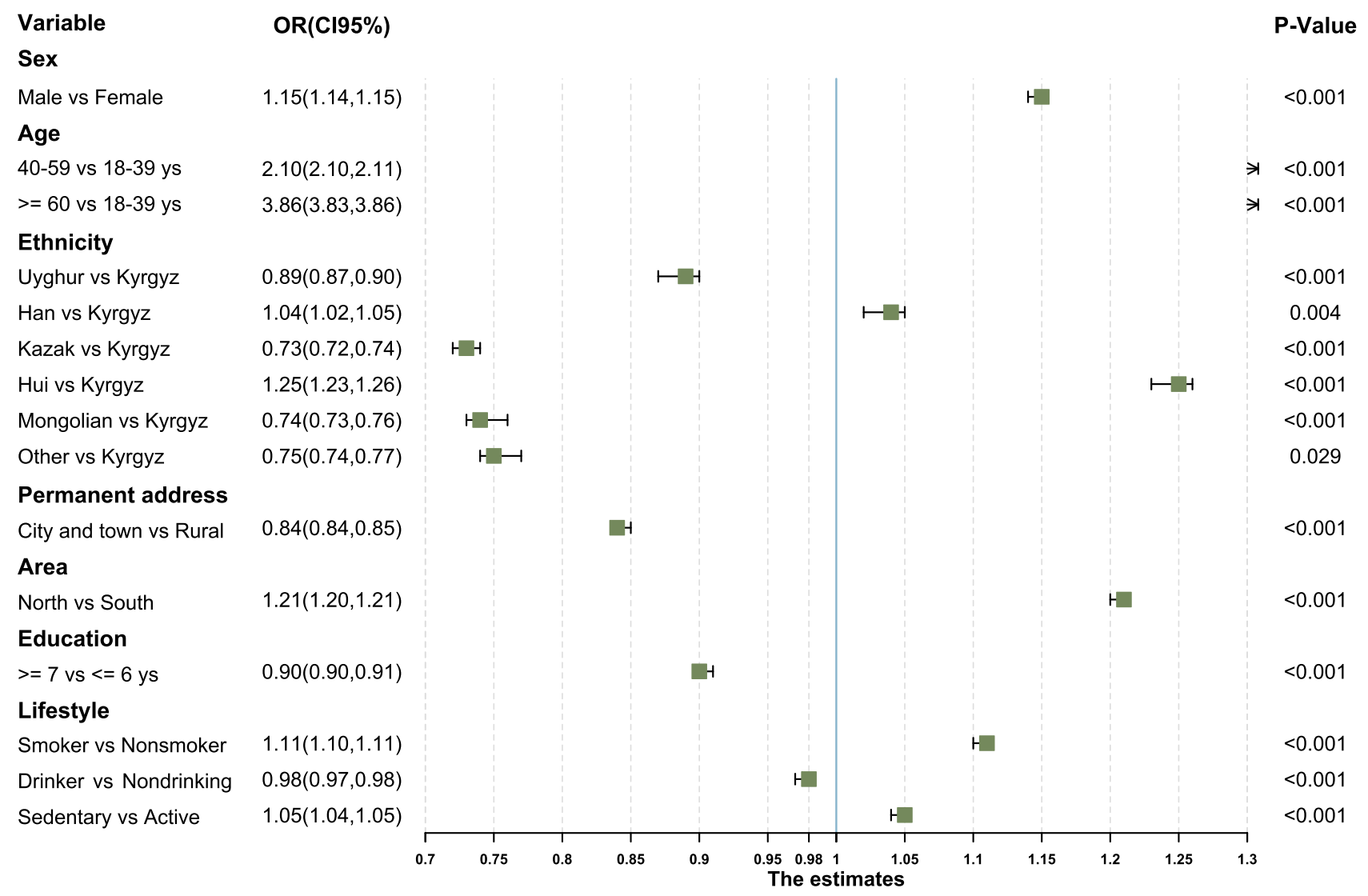

Figure 6 Odds Ratios and $95 \% \mathrm{Cls}$ for the Associations of Elevated-FBG with population and health behavior characteristics. The arrows in the figure indicate that the range of corresponding values exceed the plotting area.

due to the cold climate in the north. Outdoor exercise was less in the North than in the South, which was consistent with the results of previous studies on MetS of North and South residents. ${ }^{11}$ Southerners were more likely to develop low-HDL-C than Northern residents. Kazakh, Mongolian with probably anti-lipid genes are mostly living in northern Xinjiang to contribute to the above results.

From the perspective of educational background, the prevalence of MetS in group with $\geq 7$ years of educational experience was higher than that in those $\leq 6$ years of education. However, after adjusting for ages, ethnic areas and living habits, the risk was lower in $\geq 7$ years of education than that in $\leq 6$ years education group. Previous studies showed that higher level of education had a higher score of health literacy. ${ }^{39}$ The results of this study showed that the rate of smoking and drinking was higher, and the rate of regular exercise was lower in $\geq 7$ years of education group. Through the analysis of various components of metabolic diseases, the risk of Low-HDL-C, Elevated-BP was higher in subjects of $\leq 6$ years education, and the risk of central obesity, high-TG and high-FBG was lower in $\geq 7$ years of education group. The possible reasons are still unknown.

Interestingly, in this study, there were inconsistencies among the results of this big data analysis of unhealthy lifestyles such as smoking, drinking and lack of exercise. People who smoked, drank, and took regular exercise had a lower prevalence of MetS than that in non-smoking, nondrinking people, and those who did not take regular exercise . Through the multivariate analysis, the risk of MetS in the smoking and drinking populations was higher than that in non-smokers and non-drinkers, indicating that the risk of MetS was greatly affected by other factors, such as gender, ages, exercise, etc. In summary, the relationship between lifestyle and MetS is not fully established yet. This may be caused by the complex compositions of subgroups within the big comprehensive population.

Some ethnic groups, such as Kyrgyz, Mongolian and Kazak may have the ability to resist risk factors of MetS due probably to specific genetic factors. Further exploration of genetic contribution to MetS is warranted to clarify the relationship between MetS and genetic background of Kazakh, Mongolian and Kyrgyz in order to 


\section{Variable}

Sex

Male vs Female

Age

40-59 vs $18-39$ ys

$>=60$ vs $18-39$ ys

Ethnicity

Uyghur vs Kyrgyz

Han vs Kyrgyz

Kazak vs Kyrgyz

Hui vs Kyrgyz

Mongolian vs Kyrgyz

Other vs Kyrgyz

Permanent address

City and town vs Rural

Area

North vs South

Education

$>=7$ vs $<=6$ ys

Lifestyle

Smoker vs Nonsmoker

Drinker vs Nondrinking

Sedentary vs Active

OR(C195\%)

P-Value

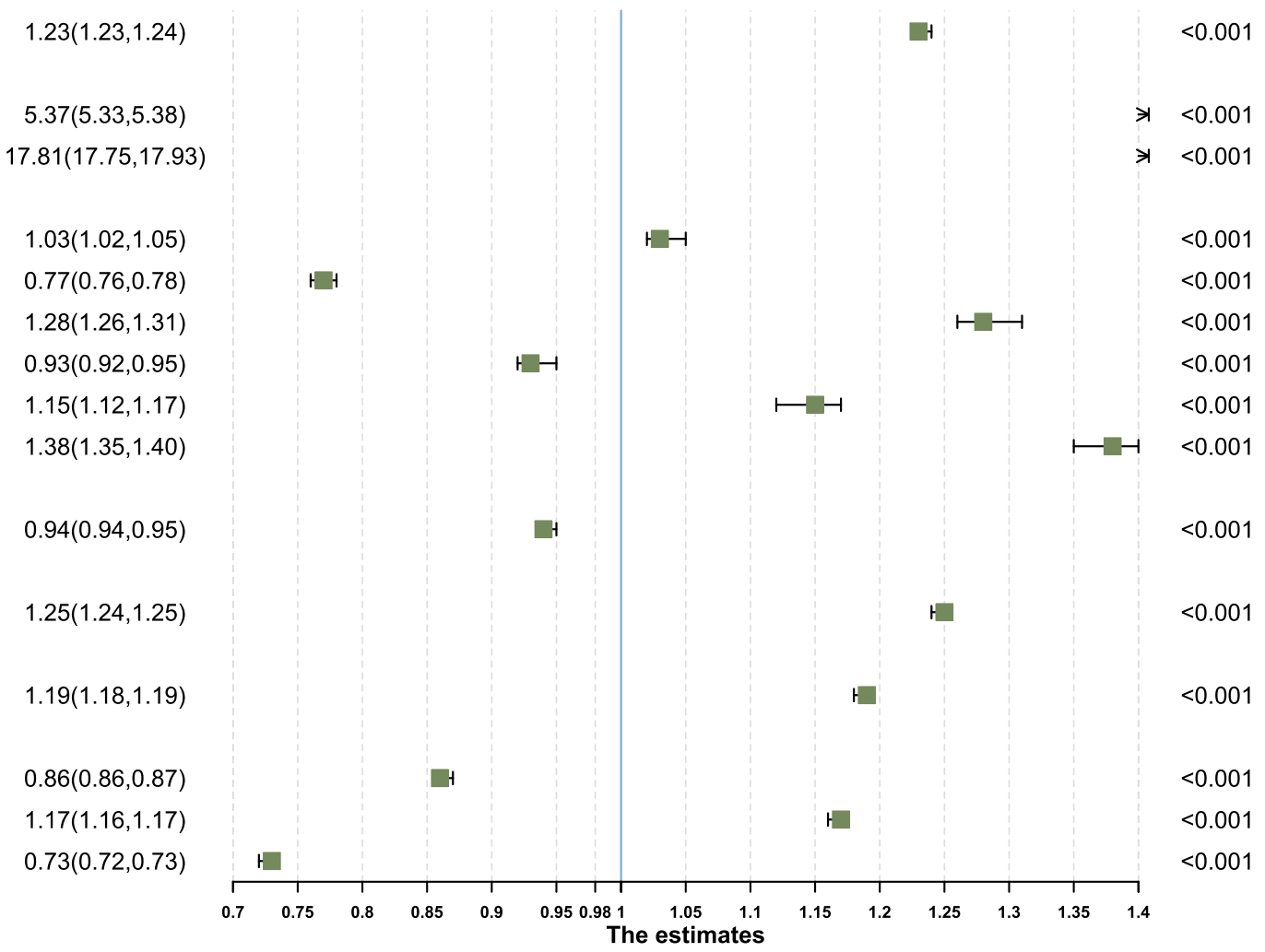

Figure 7 Odds Ratios and $95 \% \mathrm{Cls}$ for the Associations of Elevated-BP with population and health behavior characteristics. The arrows in the figure indicate that the range of corresponding values exceed the plotting area.

explore the mechanism of MetS occurrence and development.

\section{Conclusion}

Both genetic and environmental factors affect the prevalence and development of MetS. The prevalence of MetS in Kazakh, Mongolian and Kyrgyz was less even though the proportion of risk factors and the rate of unhealthy lifestyles were higher. The regular relationship between lifestyle and common risk factors was established in this large-scale comprehensive multiethnic population analysis. In addition, the prevention of central obesity was strongly recommended, especially in $\geq$ middle-aged women. Han and Hui should pay particular attention to hyperglycemia; northern Xinjiang residents should be specially encouraged to take more exercise, and urban residents should carefully restrict their alcohol consumption.

\section{Abbreviations}

MetS, metabolic syndrome; WC, waist circumference; SBP, DBP, systolic-diastolic blood pressure; TC, total cholesterol; TG, triglyceride; HDL-C, high-density lipoprotein; LDL-C, low-density lipoprotein; FBG, fasting blood glucose; ATP III, modified Adults Treatment Panel.

\section{Acknowledgments}

We thank the staff from Health Commission of Xinjiang Autonomous Region and 1315 medical institutions of local health Centers for their collection of data and blood samples. We also thank all the study participants for their contributions. Project supported by the National Natural Science Foundation of China (81960608) and Xinjiang Science and Technology Department Set Up the Project (2018E02057).

\section{Disclosure}

The authors report no conflicts of interest in this work.

\section{References}

1. Grundy SM, Cleeman JI, Daniels SR, et al. Diagnosis and management of the metabolic syndrome: an American Heart Association/ National Heart, Lung, and Blood Institute scientific statement. Curr Opin Cardiol. 2006;21(1):1-6. doi:10.1097/01.hco.0000200416.65 $370 . \mathrm{a} 0$ 
2. Lekoubou A, Ovbiagele B, Markovic D, Sanossian N, Towfighi A. Age, sex, and race/ethnic temporal trends in metabolic syndrome prevalence among individuals with myocardial infarction or stroke in the United States. J Neurol Sci. 2017;376:24-28. doi:10.1016/j.jns.2017.01.073

3. Moore JX, Chaudhary N, Akinyemiju T. Metabolic syndrome prevalence by race/ethnicity and sex in the United States, National Health and Nutrition Examination Survey, 1988-2012. Prev Chronic Dis. 2017;14:E24. doi:10.5888/pcd14.160287

4. Imitrijevic Z, Jovanovic A, Cvetkovic M, Vrecic T, Kostic E, Mitic B. Associations of cardiovascular and all-cause mortality with metabolic syndrome in hemodialysis patients: a prospective single-center study. Medicina (Kaunas, Lithuania). 2019;55(10).

5. ON S, OD L. Metabolic syndrome: a closer look at the growing epidemic and its associated pathologies. Obesity Rev. 2015;16 (1):1-12. doi:10.1111/obr.12229

6. Hirode G, Wong RJ. Trends in the prevalence of metabolic syndrome in the United States, 2011-2016. JAMA. 2020;323(24):2526-2528. doi:10.1001/jama.2020.4501

7. Anand SS, Yi Q, Gerstein H, et al. Relationship of metabolic syndrome and fibrinolytic dysfunction to cardiovascular disease. Circulation. 2003;108(4):420-425. doi:10.1161/01.CIR.0000080884.27358.49

8. Jaspers Faijer-Westerink H, Kengne AP, Meeks KAC, Agyemang C. Prevalence of metabolic syndrome in sub-Saharan Africa: a systematic review and meta-analysis. Nutri Metabol Cardiovas Dis. 2020;30(4):547-565. doi:10.1016/j.numecd.2019.12.012

9. Cameron AJ, Magliano DJ, Zimmet Z, Welborn T, Shaw JE. The metabolic syndrome in Australia: prevalence using four definitions. Diabetes Res Clin Pract. 2007;77(3):471-478. doi:10.1016/j. diabres.2007.02.002

10. Herningtyas H, Ng TS. Prevalence and distribution of metabolic syndrome and its components among provinces and ethnic groups in Indonesia. BMC Public Health. 2019;19(1):377. doi:10.1186/ s12889-019-6711-7

11. Li R, Li W, Lun Z, et al. Prevalence of metabolic syndrome in Mainland China: a meta-analysis of published studies. BMC Public Health. 2016;16(1):296. doi:10.1186/s12889-016-2870-y

12. Guo H, Gao X, Ma R, et al. Prevalence of metabolic syndrome and its associated factors among multi-ethnic adults in rural areas in Xinjiang, China. Sci Rep. 2017;7(1):17643. doi:10.1038/s41598-017-17870-5

13. W X, Howell D, Tang L, et al. Comparative study on prevalence of metabolic syndrome based on three criteria among adults in Zhejiang province, China: an observational study. BMJ Open. 2020;10(4): e035216. doi:10.1136/bmjopen-2019-035216

14. Du Z, Xing L, Liu S, et al. Prevalence and determinants of metabolic syndrome based on three definitions in rural northeast China. Public Health Nutr. 2020;1-8.

15. Li H, Guo M, An Z, et al. Prevalence and risk factors of metabolic associated fatty liver disease in Xinxiang, China. Int $J$ Environ Res Public Health. 2020;17:6.

16. Zhang Z, Fan S, Xue Z, et al. Evaluation of the appropriate predictive contributor and diagnostic threshold for the cardio-metabolic syndrome in Chinese Uyghur adults. BMC Public Health. 2019;19 (1):613. doi:10.1186/s12889-019-6803-4

17. Zhu Y, Zheng H, Zou Z, et al. Related factors in chinese children and adolescents: analysis from a Chinese National Study. $J$ Atheroscler Thromb. 2020;27(6):534-544. doi:10.5551/jat.50591

18. L Y, Mai Z, Zhou S, et al. Prevalence of metabolic syndrome in China: an up-dated cross-sectional study. PLoS One. 2018;13(4): e0196012. doi:10.1371/journal.pone.0196012

19. Appropriate body-mass index for Asian populations and its implications for policy and intervention strategies. Lancet (London, England). 2004;363(9403):157-163. doi:10.1016/S0140-6736(03)15268-3.

20. Deurenberg P, Deurenberg-Yap M, Guricci S. Asians are different from Caucasians and from each other in their body mass index/body fat per cent relationship. Obesity Rev. 2002;3(3):141-146. doi:10.1046/j.1467-789X.2002.00065.x
21. Du Z, Xing L, Liu S, et al. Prevalence and determinants of metabolic syndrome based on three definitions in rural northeast China. Public Health Nutr. 2020;1-8. doi:10.1017/S1368980019004166

22. Iqbal SP, Ramadas A, Fatt QK, et al. Relationship of sociodemographic and lifestyle factors and diet habits with metabolic syndrome (MetS) among three ethnic groups of the Malaysian population. PLoS One. 2020;15(3):e0224054. doi:10.1371/journal.pone.0224054

23. Qin L, Luo B, Gao F, Feng X, Liu J. Effect of exposure to famine during early life on risk of metabolic syndrome in adulthood: a meta-analysis. J Diabetes Res. 2020;2020:3251275. doi:10.1155/2020/3251275

24. Tan Andrew KG, Dunn RA, Yen ST. Ethnic disparities in metabolic syndrome in malaysia: an analysis by risk factors. Metab Syndr Relat Disord. 2011;9(6):441-451. doi:10.1089/met.2011.0031

25. Yim E, Lee K, Park I, Lee S. The prevalence of metabolic syndrome and health-related behavior changes: the Korea National Health Examination Survey. Healthcare (Basel, Switzerland). 2020;8:2.

26. KG A, RH E, SM G, et al. Harmonizing the metabolic syndrome: a joint interim statement of the International Diabetes Federation Task Force on Epidemiology and Prevention; National Heart, Lung, and Blood Institute; American Heart Association; World Heart Federation; International Atherosclerosis Society; and International Association for the Study of Obesity. Circulation. 2009;120(16):1640-1645.

27. Leys C, Klein O, Dominicy Y, Ley C. Detecting multivariate outliers: use a robust variant of the Mahalanobis distance. J Exp Soc Psychol. 2018;74:150-156. doi:10.1016/j.jesp.2017.09.011

28. Saklayen MGS. The Global Epidemic of the Metabolic Syndrome. Curr Hypertens Rep. 2018;20(2):12. doi:10.1007/s11906-018-0812-Z

29. Zhang Y-Y, Fu Z-Y, Wei J, et al. A LIMA1 variant promotes low plasma LDL cholesterol and decreases intestinal cholesterol absorption. Science (New York, NY). 2018;360(6393):1087-1092. doi:10.1126/science.aao6575

30. Guo Y, Chen C, Jin X, et al. Autosomal DIPs for population genetic structure and differentiation analyses of Chinese Xinjiang Kyrgyz ethnic group. Sci Rep. 2018;8(1):11054. doi:10.1038/s41598-018-29010-8

31. Mei T, Zhang L-P, Liu Y-S, et al. 24 Y-chromosomal STR haplotypic structure for Chinese Kazak ethnic group and its genetic relationships with other groups. Int J Legal Med. 2016;130(5):1199-1201. doi:10.1007/s00414-016-1331-6

32. Guo Y, Chen C, Xie T, et al. Forensic efficiency estimate and phylogenetic analysis for Chinese Kyrgyz ethnic group revealed by a panel of 21 short tandem repeats. Royal Society Open Science. 2018;5(6):172089. doi:10.1098/rsos. 172089

33. Yao Y. Different matrilineal contributions to genetic structure of ethnic groups in the silk road region in china. Mol Biol Evol. 2004;21(12):2265-2280. doi:10.1093/molbev/msh238

34. Yu S, Guo X, Yang H, Zheng L, Sun Y. An update on the prevalence of metabolic syndrome and its associated factors in rural northeast China. BMC Public Health. 2014;14(877).

35. Fujimoto WY, Bergstrom RW, Boyko EJ, et al. Type 2 diabetes and the metabolic syndrome in Japanese Americans. Diabetes Res Clin Pract. 2000;S73-76.

36. Oh SS, Kim W, Han K-T, et al. Alcohol consumption frequency or alcohol intake per drinking session: which has a larger impact on the metabolic syndrome and its components? Alcohol (Fayetteville, NY). 2018;71:15-23. doi:10.1016/j.alcohol.2018.01.005

37. Uemura H, Katsuura-Kamano S, Yamaguchi M, et al. Serum hepatic enzyme activity and alcohol drinking status in relation to the prevalence of metabolic syndrome in the general Japanese population. PLoS One. 2014;9(4):e95981. doi:10.1371/journal.pone.0095981

38. Wakabayashi I. Frequency of heavy alcohol drinking and risk of metabolic syndrome in middle-aged men. Alcohol Clin Exp Res. 2014;38(6):1689-1696. doi:10.1111/acer.12425

39. Moore JX, Chaudhary N, Akinyemiju T. Metabolic syndrome prevalence by race/ethnicity and sex in the United States, National Health and Nutrition Examination Survey, 1988-2012. Prev Chronic Dis. 2017;14:E24. 


\section{Publish your work in this journal}

Diabetes, Metabolic Syndrome and Obesity: Targets and Therapy is an international, peer-reviewed open-access journal committed to the rapid publication of the latest laboratory and clinical findings in the fields of diabetes, metabolic syndrome and obesity research. Original research, review, case reports, hypothesis formation, expert opinion and commentaries are all considered for publication. The manuscript management system is completely online and includes a very quick and fair peer-review system, which is all easy to use. Visit http://www.dovepress.com/testimonials.php to read real quotes from published authors.

Submit your manuscript here: https://www.dovepress.com/diabetes-metabolic-syndrome-and-obesity-targets-and-therapy-journal 